\title{
Injúrias dentárias traumáticas em crianças e adolescentes vítimas de violência periciadas no Instituto Médico Legal de Feira de Santana, Bahia
}

\section{Traumatic dental injuries in children and adolescents victims of violence examined at the Institute of Forensic Medicine of Feira de Santana, Bahia}

Mona Lisa Cordeiro Asselta da Silva* Jamilly de Oliveira Musse ${ }^{* *}$

André Henrique do Vale de Almeida ${ }^{* * *}$ Jeidson Antônio Morais Marques ${ }^{* * *}$ Maria Conceição Oliveira Costa****

\section{Resumo}

Objetivo: traçar o perfil epidemiológico de crianças e adolescentes que apresentaram como fator etiológico do traumatismo dentário a violência física. Sujeitos e método: estudo de casuística em crianças (0-11) e adolescentes (12-18) vítimas de violência submetidas a exame de lesão corporal, periciadas por peritos odontolegais no IML de Feira de Santana, Bahia, utilizando dados secundários, no período de $1^{\circ}$ de janeiro de 2007 a 31 de dezembro de 2014. Resultados: no período entre 2007 e 2014, foram periciados, por odontolegistas, no Instituto Médico Legal (IML) de Feira de Santana, Bahia, 107 casos de violência física contra crianças e adolescentes. Desses, 39,2\% apresentaram lesões dentárias, todas provocadas por instrumento contundente; os anos de 2011 e 2012 tiveram o maior número de vitimados (21,5\%); a maior prevalência de traumatismo dentário ocorreu em 2011 (20,0\%); 57 (53,3\%) vítimas eram do sexo feminino; $86(80,4 \%)$ pertenciam à faixa etária adolescente (12 a 18 anos); com relação à cor de pele da vítima, 76 (76,8\%) eram faiodermas; $36(83,7 \%)$ agressores não eram membros da família; $37(50,7 \%)$ vítimas foram agredidas na rua; 33 (32,7\%) vítimas apresentaram duas lesões e, dessas, 10 (30,3\%) foram de traumatismo dentário; o braço foi o meio mais utilizado para provocar as injúrias dentais traumáticas. Conclusão: a maior parte dos vitimados pela violência física que sofreram traumatismo dentário era do sexo masculino, adolescente e faioderma. Os responsáveis pelas agressões, na maioria dos casos, não tinha vínculo familiar com a vítima e as agressões ocorreram em maior prevalência na rua. As vítimas que apresentaram duas lesões foram as que sofreram o maior número de traumatismo dentário sendo na maioria dos casos provocados pelas mãos, com socos e murros.

Palavras-chave: Criança. Odontólogos. Traumatismos dentários. Violência.

Cirurgiã-dentista formada pela Universidade Estadual de Feira de Santana (UEFS). Mestranda do Programa de Pós-Graduação em Saúde Coletiva da UEFS. Aluna do curso de Especialização em Odontologia Legal da Marinha.

Cirurgiã-dentista formada pela Universidade Estadual de Feira de Santana. Mestra e doutora em Ciências Odontológicas pela Universidade de São Paulo. Professora do Departamento de Saúde/UEFS, atuando na Área de Saúde Coletiva. Perita Odontolegista no Departamento de Polícia Técnica da Bahia/IML. Enfermeiro formado pela Universidade Estadual de Feira de Santana. Mestre em Saúde Coletiva pelo Programa de Pós-Graduação em Saúde Coletiva da UEFS. Doutorando em Epidemiologia em Saúde Pública pela Escola Nacional de Saúde Pública Sergio Arouca - Fiocruz.

Cirurgião-dentista formado pela Universidade Estadual de Feira de Santana. Mestre em Odontologia pela Universidade de São Paulo. Doutor em Odontologia Preventiva e Social pela FOA-Unesp. Professor Adjunto do Departamento de Saúde/UEFS.

Médica formada pela Universidade Federal do Pará. Mestre e doutora pela Unifesp/EPM, com pós-doutorado pela UQAM. Professora Titular Pleno do Departamento de Saúde da Universidade Estadual de Feira de Santana. Coordenadora do Núcleo de Estudos e Pesquisas na Infância e Adolescência da NNEPA/UEFS. 


\section{Introdução}

O traumatismo facial é o mais prevalente dentre as inúmeras lesões consequentes de queda, acidente de trânsito e violência. Isso se deve ao fato de ser a parte do corpo mais exposta e menos protegida. $\mathrm{Na}$ maioria das vezes, o trauma facial está associado ao traumatismo de dentes, e as pessoas jovens são mais susceptíveis por serem mais ativas ${ }^{1}$.

As injúrias dentais traumáticas são consideradas um sério problema de saúde em crianças e adolescentes, com elevada prevalência ${ }^{2}$. Pesquisas apontam que, atualmente, os traumas dentais superam a incidência de lesões de cárie e de doença periodontal nessa população $0^{3}$. A consequência dos traumas nessa região, independente dos dentes decíduos ou permanentes, na maioria dos casos, é a intervenção endodôntica ou até mesmo a perda da unidade atingida, merecendo por parte do cirurgião-dentista uma melhor atenção ${ }^{2}$.

$\mathrm{Na}$ dentição decídua, o traumatismo dentário acarreta tanto perda do elemento dentário quanto danos à dentição permanente. Estudos mostram que as crianças são mais susceptíveis às injúrias dentais e que os elementos dentários mais afetados são os incisivos centrais superiores ${ }^{4}$.

Ao se tratar da dentição permanente, as consequências dessas injúrias são os tratamentos endodônticos com posterior reabilitação protética (coroa) e, na pior hipótese, a depender do tipo de trauma sofrido, da urgência e qualidade do serviço prestado, a exodontia da unidade atingida se torna necessária. Os elementos dentários mais suscetíveis são os incisivos centrais superiores, seguidos dos inferiores ${ }^{5}$.

É interessante ressaltar que, mesmo quando observados padrões de trauma dentário semelhante, o tratamento de pacientes de idades diferentes varia e o plano de tratamento deve levar em conta o paciente, os pais e o estágio de dentição em que a vítima se encontra, lembrando sempre que a criança não é apenas um adulto em miniatura, mas tem suas peculiaridades que devem ser respeitadas no momento do atendimento ${ }^{6}$.

Cavalcanti et al. $^{7}$ (2012) observaram, em seu estudo sobre traumatismos maxilofaciais em crianças e adolescentes, que $10,2 \%$ dos pacientes atendidos sofreram traumatismo dentário, desses, 71,9\% pertenciam ao sexo masculino. Ainda nesse estudo, a agressão física aparece como o quarto fator etiológico mais prevalente em relação aos traumas, o que merece, mais uma vez, atenção redobrada por parte do cirurgião-dentista, já que essa é sua área de atuação profissional, colocando-o em posição privilegiada para casos suspeitos ou confirmados da violência.

A violência com crianças e adolescentes tem sido estudada por diferentes autores nos últimos anos e, ainda assim, é um tema que está longe de ser esgotado. Os principais agressores são os pais ou res- ponsáveis legais, ou seja, as pessoas que, por lei, deveriam proteger. Nesse sentido, o cirurgião-dentista tem o dever ético, legal e social de fazer a notificação e a denúncia dos casos que tenha conhecimento dentro da sua atividade laboral, sendo, inclusive, "protegido" pelo seu código de ética sobre a necessidade da quebra do sigilo profissional. No entanto, existe uma lacuna nas identificações e notificações desses casos pelos profissionais de saúde ${ }^{8}$. Muitos desses profissionais não sabem identificar a violência ou, mesmo fazendo esse diagnóstico diferencial, não têm conhecimento do caminho a ser seguido para a notificação do caso, isso acontece devido à deficiência de abordagem desse tema na graduação e por desconhecimento acerca das obrigações legais.

Nesse contexto, o objetivo deste estudo foi traçar o perfil epidemiológico de crianças e adolescentes que apresentaram como fator etiológico do traumatismo dentário a violência física.

\section{Sujeitos e método}

Este é um estudo de casuística em crianças (0-11) e adolescentes (12-18) vítimas que foram de violência submetidas a exame de lesão corporal e periciadas por perito odontolegal no IML de Feira de Santana, Bahia, utilizando dados secundários, no período de $1^{\circ}$ de janeiro de 2007 a 31 de dezembro de 2014.

A presente pesquisa foi aprovada pelo Comitê de Ética em Pesquisa em Seres Humanos da Universidade Estadual de Feira de Santana, pelo Protocolo 151/2008 (Certificado de Apresentação para Apreciação Ética 0150.0.059.000-08).

Foi utilizada para a análise dos dados a técnica de estatística descritiva que envolveu distribuições absolutas e percentuais. Para a análise bivariada, foram utilizados os testes do Qui-Quadrado de Pearson e Exato de Fischer. Foram utilizados os programas estatísticos StatisticalPackage for Social Science for Windows 9.0 (SPSS) e o OpenEpi.

\section{Resultados}

No período de 2007 a 2014, foram periciados 107 casos de violência física com crianças e adolescentes pelos odontolegistas do Instituto Médico Legal (IML) de Feira de Santana, Bahia. Dos casos estudados, $39,2 \%$ apresentaram lesões dentárias, todas provocadas por instrumento contundente.

Os anos de 2011 e 2012 tiveram o maior número de vitimados $(\mathrm{n}=23)$ e o maior número de traumatismo dentário ocorreu no ano de 2011 (n=8) (Figura 1). 


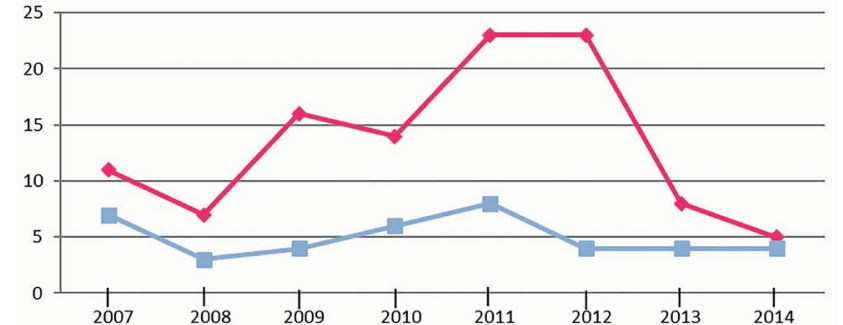

Figura 1 - Distribuição ano a ano dos casos de violência e dos traumas dentários em crianças e adolescentes vítimas periciados no IML de Feira de Santana, Bahia, 2007-2014

Fonte: dados da pesquisa.
No tocante às características sociodemográficas, observou-se que $53,3 \%$ das vítimas de violência eram do sexo feminino e a maioria $(80,4 \%)$ pertencia à faixa etária adolescente, de 12 a 18 anos de idade. Quanto à cor da pele, $76,8 \%$ eram faiodermas e $17,2 \%$ melanodermas. Com relação às características da agressão, 83,7\% dos agressores não eram membros da família, $50,7 \%$ das vítimas foram agredidas na rua, $26,0 \%$ foram agredidas em suas casas e 19,2\% na escola. Nesse grupo de variáveis, não foi encontrada associação significativa com o desfecho (traumas dentários) (Tabela 1).

Tabela 1 - Descrição das características sociodemográficas e da agressão de crianças e adolescentes vítimas de violência e análise bivariada conforme o traumatismo dentário, Feira de Santana, Bahia, 2007-2014

\begin{tabular}{|c|c|c|c|c|c|}
\hline \multirow{2}{*}{ Variáveis } & \multirow{2}{*}{$\mathrm{N}$} & \multirow{2}{*}{$\%$} & \multicolumn{2}{|c|}{ Traumatismo dentário } & \multirow{2}{*}{$p$} \\
\hline & & & $\mathrm{n}$ & $\%$ & \\
\hline \multicolumn{6}{|c|}{ Características sociodemográficas } \\
\hline \multicolumn{6}{|l|}{$\operatorname{Sexo}(N=107)$} \\
\hline Feminino & 57 & 53,3 & 19 & 33,3 & \multirow{2}{*}{0,355} \\
\hline Masculino & 50 & 46,7 & 21 & 42,0 & \\
\hline \multicolumn{6}{|l|}{ Faixa-etária (N=107) } \\
\hline Crianças & 21 & 19,6 & 8 & 38,1 & \multirow{2}{*}{0,940} \\
\hline Adolescentes & 86 & 80,4 & 32 & 37,2 & \\
\hline \multicolumn{6}{|l|}{ Cor da pele $(\mathrm{N}=99)$} \\
\hline Leucoderma & 6 & 6,1 & 1 & 16,7 & \multirow{3}{*}{0,553} \\
\hline Faioderma & 76 & 76,8 & 28 & 36,8 & \\
\hline Melanoderma & 17 & 17,2 & 7 & 41,2 & \\
\hline \multicolumn{6}{|l|}{ Características da agressão } \\
\hline \multicolumn{6}{|l|}{ Agressor $(\mathrm{N}=43)$} \\
\hline Familiar & 7 & 16,3 & 1 & 14,3 & \multirow{2}{*}{0,180} \\
\hline Não familiar & 36 & 83,7 & 18 & 50,0 & \\
\hline \multicolumn{6}{|l|}{ Local da agressão $(\mathrm{N}=73)$} \\
\hline Escola & 14 & 19,2 & 8 & 57,1 & \multirow{4}{*}{0,093} \\
\hline Casa & 19 & 26,0 & 4 & 21,1 & \\
\hline Rua & 37 & 50,7 & 12 & 32,4 & \\
\hline Outros & 3 & 4,1 & - & - & \\
\hline
\end{tabular}

Fonte: dados da pesquisa.

Na Tabela 2, em relação às características das lesões, foi observado que as ocorrências na mesma vítima de duas lesões $(32,7 \%)$, de uma lesão $(29,7 \%)$ e de três lesões $(16,8 \%)$ foram as mais frequentes nas vítimas. Em relação ao instrumento utilizado, braço $(74,7 \%)$, pés $(8,4 \%)$ e moto $(5,3 \%)$ foram os mais prevalentes. As duas variáveis foram estatisticamente significantes com os traumas dentários. 
Tabela 2 - Descrição das características das lesões de crianças e adolescentes vítimas de violência e análise bivariada conforme o traumatismo dentário, Feira de Santana, Bahia, 2007-2014

\begin{tabular}{|c|c|c|c|c|c|}
\hline \multirow{2}{*}{ Características das lesões } & \multirow{2}{*}{$N$} & \multirow{2}{*}{$\%$} & \multicolumn{2}{|c|}{ Traumatismo dentário } & \multirow{2}{*}{$p$} \\
\hline & & & $\mathrm{n}$ & $\%$ & \\
\hline \multicolumn{6}{|l|}{ Número de lesões (N=101) } \\
\hline 1 & 30 & 29,7 & 6 & 20,0 & \multirow{6}{*}{0,019} \\
\hline 2 & 33 & 32,7 & 10 & 30,3 & \\
\hline 3 & 17 & 16,8 & 8 & 47,1 & \\
\hline 4 & 8 & 7,9 & 5 & 62,5 & \\
\hline 5 & 5 & 5,0 & 4 & 80,0 & \\
\hline$\geq 6$ & 8 & 7,9 & 5 & 62,5 & \\
\hline \multicolumn{6}{|c|}{ Exemplo de instrumento utilizado $(\mathrm{N}=95)$} \\
\hline Mochila & 1 & 1,1 & 1 & 100,0 & \multirow{7}{*}{0,033} \\
\hline Pedra & 2 & 2,1 & 2 & 100,0 & \\
\hline Moto & 5 & 5,3 & 4 & 80,0 & \\
\hline Corpo & 4 & 4,2 & 3 & 75,0 & \\
\hline Carro & 4 & 4,2 & 3 & 75,0 & \\
\hline Pés & 8 & 8,4 & 2 & 25,0 & \\
\hline Braço & 71 & 74,7 & 24 & 33,8 & \\
\hline
\end{tabular}

\section{Discussão}

De acordo com os resultados encontrados na análise descritiva, os anos de 2011 e 2012 obtiveram um maior número de vitimados $(21,5 \%)$, e o maior número de traumatismo dentário ocorreu no ano de 2011 , com prevalência de $20 \%$. No estudo realizado por Cavalcanti ${ }^{9}$ (2008) em Campina Grande, foi observado que a maioria das ocorrências foi em 2005 (37,5\%), o que não vem a corroborar com este trabalho. Uma possível explicação para a divergência dos resultados pode ser o fato de que Cavalcanti pesquisou o período de 2003 a 2006.

As vítimas que apresentaram trauma dentário tiveram prevalência de $39,2 \%$. Corroborando com o estudo realizado por Oliveira et al. ${ }^{10}$ (2008), no qual foi constatado que $40,5 \%$ das vítimas de agressão foram atingidas em região dental.

Apesar de haver equilíbrio entre os gêneros, o feminino foi o mais vitimado pela violência física $(53,3 \%)$. Os estudos de Vieira et al. ${ }^{11}$ (2004) e de Brito et al. ${ }^{12}$ (2005) relatam uma maior prevalência de vítima do sexo feminino $(62,3 \%$ e $60 \%$, respectivamente), o que corrobora os resultados do presente trabalho. No entanto, no trabalho realizado por Silveira et al. ${ }^{13}$, no ano de 2005 , em que foram estudados os maus-tratos na infância e adolescência, houve uma prevalência maior de vítimas do sexo masculino (52\%). No entanto, nenhum estudo mostrou uma diferença significativa entre os sexos.

Ainda com relação ao sexo, os meninos apresentaram maior prevalência de traumatismo dentário $(52,5 \%)$ quando comparados com as meninas. Vale salientar, que as injúrias dentárias representaram $42 \%$ dentre as lesões sofridas pelas vítimas do sexo masculino. Os estudos realizados por Prata et al. ${ }^{14}$ (2000) e por Xavier et al. ${ }^{15}$ (2011) mostraram o traumatismo dentário mais prevalente no sexo masculino, com $62,91 \%$ e $74,6 \%$, respectivamente, corroborando com os resultados da presente pesquisa. Não foram encontrados estudos na literatura que trouxessem a prevalência de traumas por sexo comparado a outras lesões sofridas.

Das vítimas de violência física, $80,4 \%$ estavam na faixa etária entre 12 e 18 anos (adolescentes), dos quais, $37,2 \%$ sofreram traumatismos dentários. O estudo de Prata et al. ${ }^{14}$ (2000) aponta que a maioria dos traumas dentários ocorreu entre 7 e 11 anos de idade $(64,25 \%)$, não corroborando os achados encontrados neste estudo. Uma explicação para essa diferença de resultado poderá estar nos fatores etiológicos, já que o presente estudo utilizou como fator causador de traumatismo dentário as agressões físicas e investigou outras faixas etárias, enquanto que Prata et al. utilizaram outros fatores, sendo que a agressão/golpe apareceu como o terceiro fator etiológico mais prevalente $(15,89 \%)$ e a queda como o primeiro $(48,4 \%)$.

Com relação à cor de pele das vítimas, $76,8 \%$ eram faiodermas, dessas, 36,8\% sofreram traumatismos dentários. Um estudo feito por Guimarães e Vilella $^{16}$ (2011), que coletaram dados de crianças e adolescentes no IML de Maceió, observou que 71,6\% tinham cor de pele parda. O estudo realizado por Pimenta et al. ${ }^{17}$ (2013) evidencia que $64,2 \%$ das vítimas eram faiodermas, o que corrobora também os resultados deste estudo. Existe "consenso" na lite- 
ratura quando se refere à cor da pele de vítimas de violência física, já que estudos apontam que os mais vitimados são aqueles que se enquadram como pretos ou pardos, merecendo maior implementação de políticas públicas para esse grupo, que, por vezes, apresenta-se como mais exposto e vulnerável a sofrer esse tipo de violência.

No tocante aos agressores, o referido estudo mostrou uma maior prevalência de agressores não familiares $(83,7 \%)$, sendo que $50 \%$ desses violentadores, no momento da execução do golpe, atingiram o dente das vítimas. Silveira et al. ${ }^{13}$ (2005) observaram que os agressores, em sua maioria, eram membros da família, não corroborando o presente trabalho. No entanto, Guimarães e Vilella ${ }^{16}$ (2011) e Pimenta et al. ${ }^{17}$ (2013) encontraram, em seus respectivos trabalhos, uma maior prevalência de agressor não familiar, o que vem ao encontro dos resultados aqui apresentados. Apesar da existência de muitos estudos sobre traumatismo dentário, em que a violência se apresenta como causa dessas lesões, ainda são insuficientes as investigações quando se diz respeito aos responsáveis por provocá-las.

Com relação à localização dos violentadores no momento da agressão, a rua apareceu como local de prevalência $(50,7 \%)$, sendo que $50 \%$ das vítimas agredidas na rua tiveram traumatismo dentário. No estudo de Guimarães e Vilella ${ }^{16}$ (2011), foi notado que $17,5 \%$ das crianças e adolescentes estavam na rua no momento da agressão, no entanto, a casa da vítima foi o local mais prevalente, com $27,1 \%$, seguido de uma categoria que eles denominaram "próximo à casa", com frequência de $18,2 \%$, resultados não encontrados pelos autores deste trabalho.

Em relação à quantidade de lesões que as vítimas apresentaram no momento do exame de lesão corporal, este trabalho encontrou uma maior prevalência de vítimas com duas lesões (32,7\%), enquanto que $29,7 \%$ apresentaram apenas uma lesão. Foi observado, ainda, que as vítimas que apresentaram duas e três lesões sofreram mais traumatismo dentário, 26,3\% e 21,1\%, respectivamente. Cavalcanti $(2009)^{9}$, em seu estudo, observou que $66,7 \%$ das vítimas apresentaram uma lesão e 21,4\% apresentaram duas lesões.

O braço foi o agente mais utilizado para causar as lesões $(74,7 \%)$, dentre as quais, $33,8 \%$ causaram traumas dentários nas vítimas, corroborando com o estudo realizado por Oliveira et al. ${ }^{10}$ (2008), o qual encontrou que 58,2\% dos agentes utilizados para provocar as injúrias dentárias foram os braços e os pés. Diferentes estudos apontam o braço como principal agente responsável pela agressão física de crianças e adolescentes, pela facilidade que os agressores têm de usá-los. Tratando-se de traumatismo dentário, que é o objeto de estudo desta pesquisa, o murro ou soco, sem dúvidas, pode provocar uma frequência muito alta desse tipo de lesão.

\section{Conclusão}

A maior parte das vítimas de violência física que sofreram traumatismo dentário era do sexo masculino, adolescente e faioderma. Os responsáveis pelas agressões, na maioria dos casos, não tinham vínculo familiar com a vítima, e as agressões ocorreram em maior prevalência na rua. As vítimas que apresentaram duas lesões foram as que sofreram o maior número de traumatismo dentário, na maioria dos casos provocados pelas mãos, com socos e murros.

Conhecer o perfil epidemiológico de crianças e adolescentes que apresentaram como fator etiológico do traumatismo dentário a violência física permite ao cirurgião-dentista suspeitar com mais facilidade sobre os casos que chegam aos seus cuidados, seja em consultório particular, em USF seja em hospitais, podendo, assim, adquirir subsídios para cumprir o seu papel ético, legal e social na notificação e denúncia dos casos suspeitos e/ou confirmados de violência contra crianças e adolescentes.

\section{Abstract}

Objective: To draw the epidemiological profile of children and adolescents who presented physical violence as etiological factor of dental trauma. Subjects and method: Case study of children (0-11 years old) and adolescents (12-18 years old) victims of violence under consideration of personal injury, and examined by forensic dental experts at the Institute of Forensic Medicine (Instituto Médico Legal - IML) of Feira de Santana, Bahia, Brazil. Secondary data was used from January 1, 2007 to December 31, 2014. Results: From 2007 to 2014, forensic dentists examined 107 physical violence cases involving children and adolescents, at the IML of Feira de Santana - Bahia, Brazil. From these cases, 39.2\% presented dental injuries, all of them caused by blunt instrument. The years of 2011 and 2012 had the highest number of victims $(21.5 \%)$, whereas highest prevalence of dental trauma occurred in 2011 (20.0\%). Fifty-seven $(53.3 \%)$ victims were women and $86(80.4 \%)$ were adolescents (12-18 years old). Regarding skin color of the victim, 76 (76.8\%) were brown. Thirty-six (83.7\%) offenders were not family members and 37 (50.7\%) victims were assaulted in the street. Thirty-three (32.7\%) victims had two injuries and 10 of these $(30.3 \%)$ were dental traumas. The arm was mostly used to cause traumatic dental injuries. Conclusion: The majority of physical violence victims who suffered dental trauma were brown adolescent men. In most cases, the offenders had no bond with the family of the victim, and the higher prevalence of assaults occurred in the street. Victims who presented two injuries were the ones who suffered the highest number of dental trauma, which were mostly caused by hand punches.

Keywords: Children. Dental trauma. Dentists. Violence. 


\section{Referências}

1. Godoi MS, Basualdo A, Oliveira KC. Índice de fraturas faciais no Hospital São Vicente de Paulo em Passo Fundo RS: estudo retrospectivo de dez anos. J Oral Invest 2013; 2(2):14-9.

2. Simões FG, Leonardi DP, Barato Filho F, Ferreira EL, Fariniuk LF, Sayão SMA. Fatores etiológicos relacionados ao traumatismo alvéolo-dentário de pacientes atendidos no pronto-socorro odontológico do Hospital Universitário Cajuru. RSBO 2004; 1(1):50-5.

3. Ishida AL, Endo MS, Kitayama VS, Pavan AJ, Queiroz AF, Pavan NNO. Avulsão dentária e fatores relacionados ao prognóstico: estudo retrospectivo de 13 anos. Arquivos do MUDI 2014; 18(3):17-28.

4. Pereira AD, Boer NP, Correia TM, Lima DP, Cunha-Correia ASC. Traumatismo na dentição decídua - diagnóstico, prognóstico e acompanhamento de um caso. Arch Health Invest 2014; 3(6):14-9.

5. Ruslin M, Wolff J, Boffano P, Brand HS, Forouzanfar T. Dental trauma in association with maxillofacial fractures: an epidemiological study. Dent Traumatol 2015; 31(4):318-23.

6. Monson LA, Smith DM, Losee JE. Pediatric Facial Fractures. Ferraro's Fundamentals of Maxillofacial Surgery $2015 ; 7(2): 283-97$

7. Cavalcanti AL, Assis KM, Cavalcante JR, Xavier AFC, Aguiar YPC. Traumatismos maxilofaciais em crianças e adolescentes em Campina Grande, Paraíba, Brasil. Pesq Bras Odontoped Clin Integr 2012; 12(3):439-45.

8. Pereira LS, Costa LS, Carrijo LF, Silva GQ, Silva MB. A violência domiciliar contra crianças e adolescentes e a responsabilidade dos profissionais de saúde: uma revisão bibliográfica. Rev Edu Saúde 2015; 2(1):127-39.

9. Cavalcanti AL. Lesões no complexo maxilofacial em vítimas de violência no ambiente escolar. Ciên Saúde Coletiva 2008; $4(5): 1835-42$

10. Oliveira CMCS, Santos JS, Braseileiro BF, Santos TS. Epidemiologia dos traumatismos buco-maxilo-faciais por agressões em Aracaju/SE. Rev Cir Traumatol Buco-Maxilo-Fac 2008; 8(3):57-68.

11. Vieira D, Martins CS, Ferriani MGC, Nascimento LC. Caracterização da violência física contra crianças e adolescentes. Rev Enferm UERJ 2004; 12:306-11.

12. Brito AMM, Zanetta DMT, Mendonça RCV, Barison SZP, Andrade VAG. Violência doméstica contra crianças e adolescentes: estudo de um programa de intervenção. Ciên Saúde Coletiva 2005; 10(1):143-9.

13. Silveira JLGC, Mayrink S, Netto OBS. Maus-tratos na infância e adolescência: casuística, conhecimento e prática de cirurgiões-dentistas de Blumenau-SC. Pesq Bras Odontoped Clin Integr 2005; 5(2):119-26.

14. Prata THC, Duarte MSR, Miquilito JL, Valera MC, Araújo MAM. Etiologia e freqüência das injúrias dentárias traumáticas em pacientes do centro de traumatismos dentários da Faculdade de Odontologia de São José dos Campos - Unesp. Rev Odontol 2000; 29(1/2):43-53.

15. Xavier CB, Faria GD, Vogt BF, Collares KF, Dickel R. Estudo dos traumatismos alvéolo-dentários em pacientes atendidos em um Setor de Cirurgia e Traumatologia Buco-Maxilo-Facial. Rev Gaúcha Odontol 2011; 59(4):565-70.

16. Guimarães JATL, Vilella WV. Características da violência física e sexual contra crianças e adolescentes atendidos no IML de Maceió, Alagoas, Brasil. Cad Saude Publica 2011; 27(8):1647-153.
17. Pimenta RMC, Matos FRRO, Silva MLCA, Rodrigues AAAO, Marques JAM, Musse JO. Levantamento de lesões na região bucomaxilofacial em vítimas de violência periciadas no Instituto Médico Legal (IML) de Feira de Santana-BA, entre 2007 e 2009. Arq Odontol 2013; 49(4):154-61.

\section{Endereço para correspondência:}

Mona Lisa Cordeiro Asselta da Silva

Rua Barcelona, 136

Residencial Arthur A. Cordeiro, apart. 101

44076-720 Feira de Santana, Bahia

Telefone: (75) 92317850

E-mail: mona.cordeiro@hotmail.com

Rcebido: 16/09/15. Aceito: 22/06/16. 